\title{
Notes ouest-africaines
}



\section{Intégration régionale des villes frontalières}

AVRIL 2019 NN$^{\circ} 20$

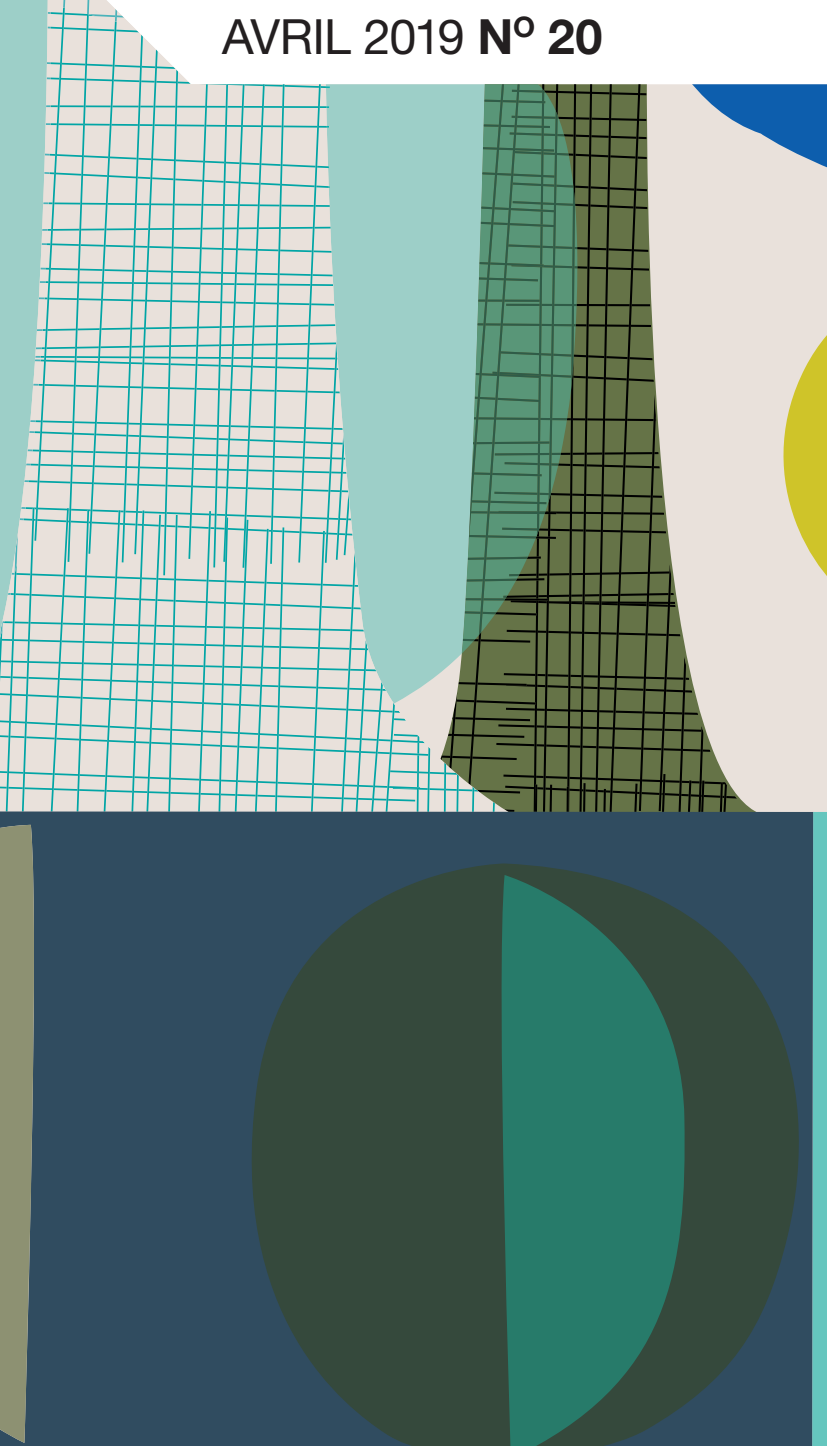

Secrétariat d

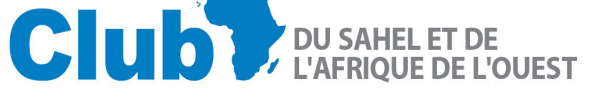





\section{INTÉGRATION RÉGIONALE DES VILLES FRONTALIÈRES \\ Collection «Villes»}

Sous la direction de Marie Trémolières et Olivier J. Walther

\section{Aussi dans cette Collection:}

"Population et morphologies des villes frontalières", $\mathbf{N}^{\circ} 21$

"Entreprises et santé dans les villes frontalières", № 22

"Accessibilité et infrastructures des villes frontalières", № 23

Secrétariat du 


\section{NOTES OUEST-AFRICAINES}

Les Notes ouest-africaines analysent les dynamiques socio-économiques, politiques et sécuritaires que traverse l'Afrique dans une perspective régionale et multidisciplinaire. Elles cherchent à stimuler la discussion, rassembler les informations et mieux anticiper les transformations en cours pour les politiques à venir. Elles visent à partager des études avec une large audience d'experts, de praticiens du développement, de décisionnaires et de lecteurs avertis. Les Notes sont disponibles en anglais et/ou en français; les résumés dans les deux langues. Initiées par le Club du Sahel et de l'Afrique de l'Ouest (CSAO) pour éclairer les enjeux ouest-africains, ces analyses sont préparées par son Secrétariat, ses membres et partenaires, les autres départements de l'OCDE, des organisations internationales et autres experts et chercheurs.

Merci de citer cet ouvrage comme suit:

OCDE/CSAO (2019), ( Intégration régionale des villes frontalières )),

Notes ouest-africaines, No20, Éditions OCDE, Paris.

https://doi.org/10.1787/fd0fe15f-fr

ISSN 2414-2026

Ce document est publié sous la responsabilité du Secrétaire général de l'OCDE. Les opinions et les interprétations qui y sont exprimées et les arguments qui y sont employés ne reflètent pas nécessairement les vues officielles des gouvernements des pays membres de l'OCDE.

Ce document et toute carte qu'il peut comprendre ne préjugent en rien du statut de tout territoire, de la souveraineté s'exerçant sur ce dernier, du tracé des frontières et limites internationales, et du nom de tout territoire, ville ou région.

Autorisé pour publication par Laurent Bossard, Directeur, Secrétariat du Club du Sahel et de l'Afrique de l'Ouest (CSAO/OCDE).

Vous êtes autorisés à copier, télécharger ou imprimer du contenu OCDE pour votre utilisation personnelle. Vous pouvez inclure des extraits des publications, des bases de données et produits multimédia de l'OCDE dans vos documents, présentations, blogs, sites Internet et matériel d'enseignement, sous réserve de faire mention de la source OCDE et du copyright. Les demandes pour usage commercial ou de traduction devront être adressées à rights@oecd.org. 


\section{Résumé}

Cette Note qui fait partie de la Collection ((Villes ), souligne la contribution des villes frontalières au processus d'intégration régionale en Afrique de l'Ouest. Six indicateurs permettent d'appréhender les dynamiques locales développées en milieu urbain, les effets de la distance sur la cohésion nationale, et l'impact des divisions territoriales au plan international dans 18 pays. Cette analyse met en évidence les obstacles qui limitent le potentiel d'intégration des villes frontalières du point de vue des échanges socio-économiques et des institutions. La Note conclut par une synthèse des politiques territorialisées pouvant être mises en place dans la région pour faciliter le développement économique et politique des villes frontalières ouest-africaines.

Mots-clés: intégration régionale, villes frontalières, Afrique de l'Ouest, analyses multidimensionnelles, politiques territorialisées

Classification JEL: O18, O21, F15, F10

\section{À propos de la collection}

Résultant d'une démographie croissante et des évolutions sociétales et économiques, l'urbanisation est l'une des transformations les plus spectaculaires de l'Afrique. Ses dynamiques et ses impacts doivent être informés, cartographiés, mesurés et projetés pour construire des options politiques soutenables. C'est l'objet de la Collection «Villes ).

\section{Le Club du Sahel et de l'Afrique de l'Ouest}

Le Club du Sahel et de l'Afrique de l'Ouest (CSAO) est une plateforme internationale indépendante. Le Secrétariat est hébergé au sein de l'Organisation de coopération et de développement économiques (OCDE). Sa mission est de promouvoir des politiques régionales à même d'améliorer le bien-être économique et social des populations ouestafricaines. Ses objectifs sont d'améliorer la gouvernance régionale de la sécurité alimentaire et nutritionnelle, et de comprendre les transformations en cours dans la région et leurs implications en matière de politiques publiques par des analyses régionales, spatiales et prospectives. Ses Membres et partenaires sont l'Autriche, la Belgique, le Canada, la Commission de la CEDEAO, le CILSS, les États-Unis, la Commission européenne, la France, le Luxembourg, la Norvège, les Pays-Bas, la Suisse et la Commission de l'UEMOA. Le CSAO a conclu des protocoles d'accord avec l'Agence du NEPAD et l'Université de Floride (Groupe de recherche sur le Sahel).

En savoir plus : www.oecd.org/csao 


\section{L'équipe et les remerciements}

L'équipe rédactionnelle et éditoriale du Secrétariat du CSAO/OCDE est composée de:

Marie Trémolières, Direction exécutive, marie.tremolieres@oecd.org Collaborations : Lia Beyeler, Freerk Boedeltje et Sylvie Letassey

Graphisme: Grand Krü Berlin/Daniel Krüger, daniel@grandkrue.de, et Martin Rümmele (cartes)

Ce travail est réalisé dans le cadre du protocole d'accord avec l'Université de Floride (Groupe de recherche sur le Sahel).

\section{La direction scientifique, la coordination des travaux sur le terrain,} la cartographie et la rédaction du rapport sont assurées par Olivier J. Walther. Dr Walther est professeur associé invité au Centre d'études africaines de l'Université de Floride, professeur associé en sciences politiques à l'Université du Sud-Danemark et consultant pour le Secrétariat du CSAO/OCDE. owalther@ufl.edu

\section{Les contributeurs externes sont:}

Hye Ryeon Jang, doctorante au département des sciences politiques de l’Université de Floride.hrjang52@ufl.edu

Michiel van Eupen, expert en modélisation spatiale, chercheur à l'institut de recherche Alterra (Université de Wageningen).michiel.vaneupen@wur.nl

Lawali Dambo, enseignant-chercheur au Département de géographie de l’Université Abdou Moumouni de Niamey (Niger). lawali.dambo@gmail.com

L'équipe du Secrétariat aimerait remercier les personnes suivantes pour leur contribution à la rédaction de ce rapport : Laurent Bossard, Philipp Heinrigs, Secrétariat du CSAO/OCDE; Paul Nugent, Isabella Soi, Wolfgang Zeller, Université d’Edimbourg; Dieudonné Kam, Brahima Cissé, CILSS; Younsa Yansambou Habibatou, REACH; Edmond Sougué;

Mohamadou Abdoul, Mamoudou Tapily, Agence allemande de coopération internationale pour le développement; Amadou Oumarou, Moustapha Koné, Université de Niamey; Jade Siu, Université de Birmingham; Laure Dumolard, Organisation mondiale de la santé ; Moussa Ismaïla Touré, Binta Diakité, Agence pour la promotion des investissements au Mali; Sarah McKune, Leonardo A. Villalón, Greg Kiker, Alioune Sow, Université de Floride; Bruce Whitehouse, Lehigh University; Armelle Choplin, Université de Paris-Est; Christine Stabell Benn, Université du Sud-Danemark; Denis Retaillé, Université de Bordeaux; Jean Rubio, Alexandra Lafont, Petia Tzvetanova, Jean Peyrony, Mission opérationnelle transfrontalière (MOT). 
Table des matières

SIGLES ET ABRÉVIATIONS

RÉSUMÉ

LE RÔLE DES VILLES FRONTALIÈRES DANS

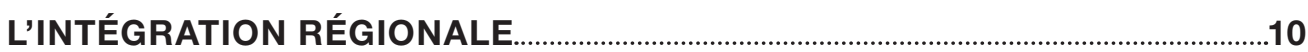

Villes, frontières et intégration régionale.................................................................10

Densité, distance et division......................................................................................12

Quelles sont les spécificités des villes frontalières ?...............................................13

Quels obstacles au développement urbain transfrontalier?..........................16

Quelles politiques de développement pour les villes frontalières ?.........17

UNE APPROCHE MULTIDIMENSIONNELLE DES

VILLES FRONTALIĖRES

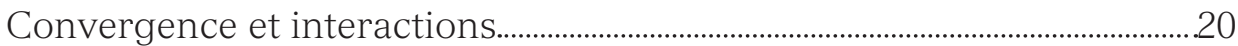

Une analyse multidimensionnelle.............................................................................22

Six indicateurs d'intégration.......................................................................................24

RÉFÉRENCES

\section{CARTES}

Carte 1.1 Régions administratives.............................................................12

Carte 1.2 L'agglomération transfrontalière de N’Djaména-

Kousséri (Tchad-Cameroun).......................................................15

\section{ENCADRÉ}

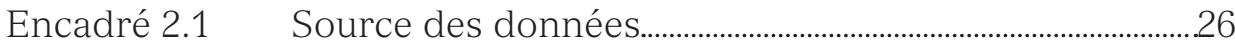

\section{GRAPHIQUES}

Graphique 1.1 Densité, distance et division.

Graphique 2.1 Convergence et interactions dans le processus d'intégration

\section{TABLEAUX}

Tableau 1.1 Indicateurs et dimensions de l'intégration

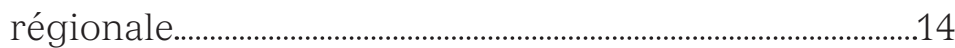

Tableau 2.1 Indicateurs relatifs aux villes frontalières..........................23 
Sigles et abréviations

CCT Conseil des collectivités territoriales

FENU Fonds d'équipement des Nations Unies

HDX Humanitarian Data Exchange

LOBI Initiative locale transfrontalière

PCTL Programme de coopération transfrontalière locale

PNUD Programme des Nations Unies pour le développement

SATI Schémas d'aménagement transfrontaliers intégrés

UEMOA Union économique et monétaire ouest-africaine

UNHCR Haut-Commissariat aux réfugiés des Nations Unies

UNOCHA Bureau de la coordination des affaires humanitaires des

Nations Unies

Cette Note s'appuie sur les données Africapolis produites par le CSAO. Elles reposent sur une approche spatiale et appliquent un critère physique (une zone bâtie en continu) et démographique (plus de 10000 habitants) pour définir une agglomération urbaine. Le terme de ville est ici utilisé au même titre que celui d'agglomération. 


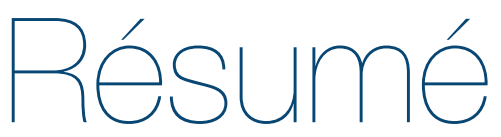

Ce rapport qui compte quatre Notes ouest-africaines (n ${ }^{\text {os }}$ 20, 21, 22 et 23) est le fruit d'une analyse systématique du rôle joué par les villes frontalières dans le processus d'intégration régionale en Afrique de l'Ouest. Sur la base d'une cartographie multidimensionnelle de 18 pays, le rapport analyse les dynamiques locales développées en milieu urbain, les effets de la distance sur la cohésion nationale, et l'impact des divisions territoriales au niveau international.

\section{Densité, distance et division}

- Au niveau local, l'étude des changements démographiques et morphologiques identifie les effets de la densité sur les villes frontalières. Elle montre que depuis le milieu du XX $\mathrm{X}^{\mathrm{e}}$ siècle, la croissance des villes frontalières est presque toujours supérieure à celle des autres villes de la région. Cette croissance rapide est particulièrement visible à moins de 50 kilomètres des frontières nationales, où sont localisés les marchés les plus dynamiques. La croissance est notamment élevée sur les frontières du Nigéria et dans le golfe de Guinée entre le Bénin et le Togo. Le rapport confirme les spécificités des villes frontalières. Vingt-sept d'entre elles forment des agglomérations transfrontalières séparées par des frontières terrestres, par des fleuves ou situées le long d'un littoral. Ces agglomérations ont émergé sans schéma d'aménagement concerté et demeurent très dépendantes les unes des autres.

- Au niveau national, les effets de la distance sur les villes frontalières sont étudiés par les indicateurs de services de santé et des entreprises formelles. La cartographie de la couverture sanitaire régionale montre la profonde hétérogénéité des espaces frontaliers ouest-africains et le potentiel encore largement inexploité d'harmonisation des politiques de santé entre pays. Celle des entreprises formelles spécialisées dans des secteurs stratégiques pour l'intégration régionale révèle une très forte concentration dans les capitales politiques et/ou économiques où se décident les politiques douanières, les stratégies d'importation et de réexportation et où sont localisées les plus grandes infrastructures de transport et de communication. Ces résultats suggèrent que le manque d'investissements publics en matière de services de santé, d'infrastructures routières et d'infrastructures éducatives dans les régions frontalières peut potentiellement poser de graves problèmes de cohésion nationale.

- Au niveau international, les effets de la division territoriale sont appréhendés par un modèle d'accessibilité développé pour rendre compte des effets de frontière sur les échanges régionaux. Les résultats montrent 
que le bassin de population des villes frontalières pourrait être $14 \%$ plus important si le passage des frontières n'avait pas d'incidence sur les flux de biens et de personnes et de 12 à $50 \%$ plus important sans poste de contrôle routier. Entre le Bénin, le Niger et le Nigéria, une analyse de l'état du réseau routier montre que le bassin de population combiné des huit villes frontalières augmenterait d'un tiers en l'absence d'attente aux frontières. La revue des postes de contrôle juxtaposés mis en œuvre ou projetés par les États ou les organisations régionales révèle en outre que la facilitation du commerce se heurte aux intérêts particuliers des fonctionnaires et des acteurs privés vivant des frictions de l'intégration régionale. En Afrique de l'Ouest en particulier, peu d'États sont aujourd'hui capables de tirer avantage de ces nouvelles structures de contrôle, dont la plupart sont non fonctionnelles.

\section{Principaux obstacles au développement urbain transfrontalier}

Le rapport confirme que les villes frontalières connaissent un déficit d'infrastructures freinant leur développement comme centres d'innovation et carrefours commerciaux régionaux. Ces contraintes s'expriment localement par un développement urbain très largement spontané et par une insuffisance des marchés, zones de stockage, voies de communication urbaines et structures médico-sociales et éducatives. Les villes frontalières peinent également de l'engorgement et du manque d'entretien des infrastructures routières et ferroviaires qui les relient au reste du pays et aux pays voisins. Le manque d'investissements productifs, socio-éducatifs et marchands, caractéristique des villes frontalières, réduit considérablement les économies d'agglomérations potentielles qui résultent de la concentration urbaine. Il renforce en outre les effets négatifs de la distance au plan national et impose de lourdes contraintes aux échanges régionaux.

Du point de vue institutionnel, le principal obstacle réside dans la faible capacité financière des collectivités locales et régionales. Bien que la décentralisation ait atteint une traduction juridique et institutionnelle suffisante, la mise à disposition des outils et des moyens financiers nécessaires à sa mise en œuvre reste limitée. La faible autonomie fiscale, le taux de recouvrement limité et la tendance à ne pas augmenter les impôts en période électorale aggravent cette situation. En conséquence, les collectivités locales manquent souvent de moyens pour mettre en place des investissements décentralisés qui permettraient d'exploiter leurs potentialités urbaines et frontalières.

\section{Options de politique}

L'importance démographique et économique des villes frontalières pour l'intégration régionale justifie que des politiques territorialisées adaptées à leurs spécificités soient mises en œuvre. 
- Les politiques de développement devraient favoriser les avantages potentiels résultant de la densité urbaine au niveau local en appuyant le développement de plans d'aménagement qui maximisent les interactions intra-urbaines. Les économies d'agglomération découlant des fortes densités urbaines permettent potentiellement de minimiser les coûts de transport et de communication, de faciliter la diffusion d'innovations et de rendre plus accessibles certains produits agricoles ou manufacturés. Elles sont cependant plus facilement exploitables par les acteurs si les villes se développent de manière planifiée et concertée de part et d'autre de la frontière. La croissance rapide de la population et l'étalement urbain observés dans les villes frontalières devraient inciter les États et leurs partenaires à investir durablement dans l'accompagnement de ces dynamiques s'ils souhaitent tirer bénéfice des économies d'agglomérations.

- Au plan national, l'enjeu des politiques de développement est de réduire la distance qui sépare les villes frontalières des autres nœuds du réseau urbain afin de minimiser les inconvénients liés à leur marginalisation géographique et de favoriser la cohésion nationale. Le développement des villes frontalières est indissociable des politiques nationales visant à projeter l'autorité et les services de l'État sur l'ensemble du territoire national.

- Au niveau international, les politiques de développement doivent continuer à réduire les frictions qui touchent les 32000 kilomètres de frontières terrestres qui divisent les États d'Afrique de l'Ouest. Cet objectif peut être atteint en facilitant la mobilité des biens, des personnes, du capital et des idées à courte et longue distance. Ainsi, les corridors de transport, les ports secs, les postes de contrôle juxtaposés, les zones de libre-échange et les pipelines actuellement implémentés par les organisations régionales et les États, devraient être davantage soutenus par les politiques de développement. 

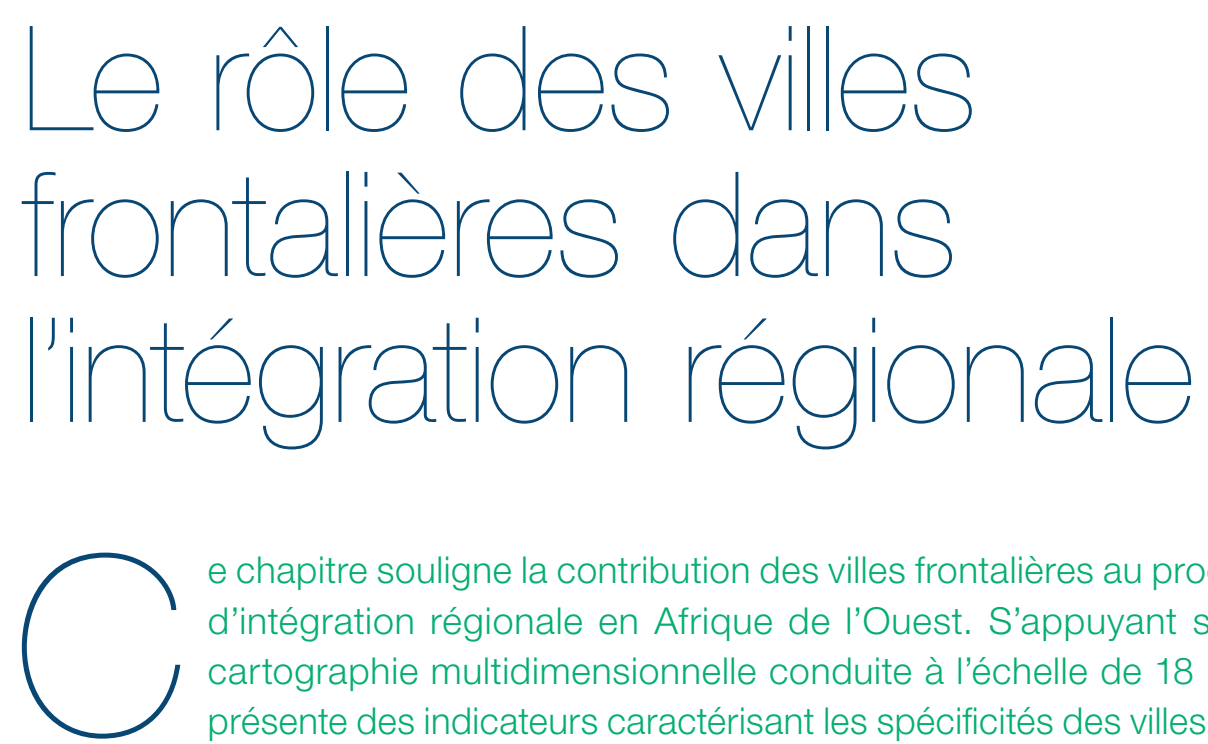

e chapitre souligne la contribution des villes frontalières au processus d'intégration régionale en Afrique de l'Ouest. S'appuyant sur une cartographie multidimensionnelle conduite à l'échelle de 18 pays, il présente des indicateurs caractérisant les spécificités des villes frontalières à l'échelle locale, nationale et internationale. II détaille ensuite les principaux obstacles auxquels elles sont confrontées du point de vue des échanges socioéconomiques et des institutions étatiques et décentralisées. II conclut par une synthèse des politiques de développement territorialisées pouvant être mises en place dans la région pour faciliter le développement économique et politique des villes frontalières ouest-africaines.

\section{Villes, frontières et intégration régionale}

L'Afrique de l'Ouest connaît un processus d'urbanisation sans précédent. Alors que moins de 30 \% de la population de la région était urbaine en 1980, près d'un habitant sur deux vivra en ville d'ici la fin de la décennie. Au Cabo Verde, au Cameroun, en Gambie, au Ghana, au Nigéria, au Sénégal et au Togo, la majorité de la population habite déjà dans un centre urbain de plus de 10000 habitants en 2015. Ce processus d'urbanisation, alimenté par une forte natalité et par d'importants mouvements migratoires, conduit à une augmentation de la taille et du nombre de villes. Pas moins de 29 villes ont dépassé le million d’habitants en 2015, dont Lagos, la plus grande ville de la région, qui compte près de 12 millions d’habitants (OCDE/CSAO, 2018). D'ici la fin des années 2010, Abidjan et Accra compteront plus de 5 millions d’habitants tandis que Bamako, Dakar, Kano et Kumasi dépasseront les 3 millions. L'augmentation rapide de la population urbaine au cours des dernières décennies se traduit également par une croissance des petites et moyennes villes. L'Afrique de l'Ouest compte ainsi 2469 agglomérations urbaines en 2015, contre 152 en 1950.

Cette évolution conduit à la formation d'un semis urbain de plus de 182 millions de personnes en 2015 dont l'une des caractéristiques principales est d'être transfrontalier. Un quart du territoire et près d'un cinquième de 
la population urbaine de l'Afrique de l'Ouest se situent en effet à moins de 50 kilomètres d'une frontière terrestre. Ces populations urbaines frontalières représentent plus de 27 millions d'habitants en 2015, réparties dans 681 villes de taille et d'importance très diverses. La présence de frontières nationales est susceptible d'avoir des effets très différents selon la situation géographique et les caractéristiques démographiques, économiques et politiques de ces villes.

La situation frontalière encourage bien évidemment le commerce à plus ou moins longue distance à partir des centres urbains. C'est le cas de Lomé, au Togo, dont la périphérie jouxte la frontière et qui dessert à la fois le Ghana voisin et les pays sahéliens grâce à ses infrastructures portuaires. D'autres villes de taille plus modeste servent également de plateforme aux échanges marchands transfrontaliers. Sur la frontière séparant le Niger du Nigéria, par exemple, Gaya, Birni N’Konni, Illela, Jibia ou Dan Issa sont des centres de collecte des produits agricoles locaux et des carrefours commerciaux pour les flux de marchandises venus des marchés mondiaux. Les différentiels législatifs, les interdictions d'importation et l'évolution des taux de change entre pays contribuent au développement souvent rapide de ces centres urbains.

La proximité à la frontière permet également de mettre en œuvre des initiatives de coopération originales, qui renforcent le processus d'intégration régionale. Les villes frontalières ne sont en effet pas seulement un maillon indispensable des flux qui traversent la région; elles forment également un noyau de vie des populations frontalières et une arène politique pour les collectivités territoriales. La proximité frontalière permet de mutualiser des infrastructures socio-économiques au plus proche des populations locales, comme le centre de santé partagé de Warokuy au Burkina Faso et Wanian au Mali. Pour d'autres centres urbains comme Dori au Burkina Faso et Téra au Niger, la situation frontalière constitue une opportunité de mettre en place des schémas d'aménagements partagés et de bénéficier d'accompagnements des organisations régionales africaines et de financements des bailleurs internationaux.

Dans ce contexte, ce rapport propose une analyse systématique de la contribution des villes frontalières au processus d'intégration régionale. S'appuyant sur une cartographie multidimensionnelle conduite à l'échelle de 18 pays (Carte 1.1), le rapport analyse les spécificités démographiques, morphologiques, sociales, économiques et politiques des villes frontalières de la région. Plusieurs indicateurs permettent d'appréhender les dynamiques locales développées en milieu urbain, les effets de la distance sur la cohésion nationale, et l'impact des divisions territoriales au plan international. Cette analyse met en évidence les principaux obstacles qui limitent le potentiel d'intégration des villes frontalières du point de vue des échanges socioéconomiques et des institutions. L'étude de ces aspects fondamentaux 


\section{Carte 1.1}

Régions administratives

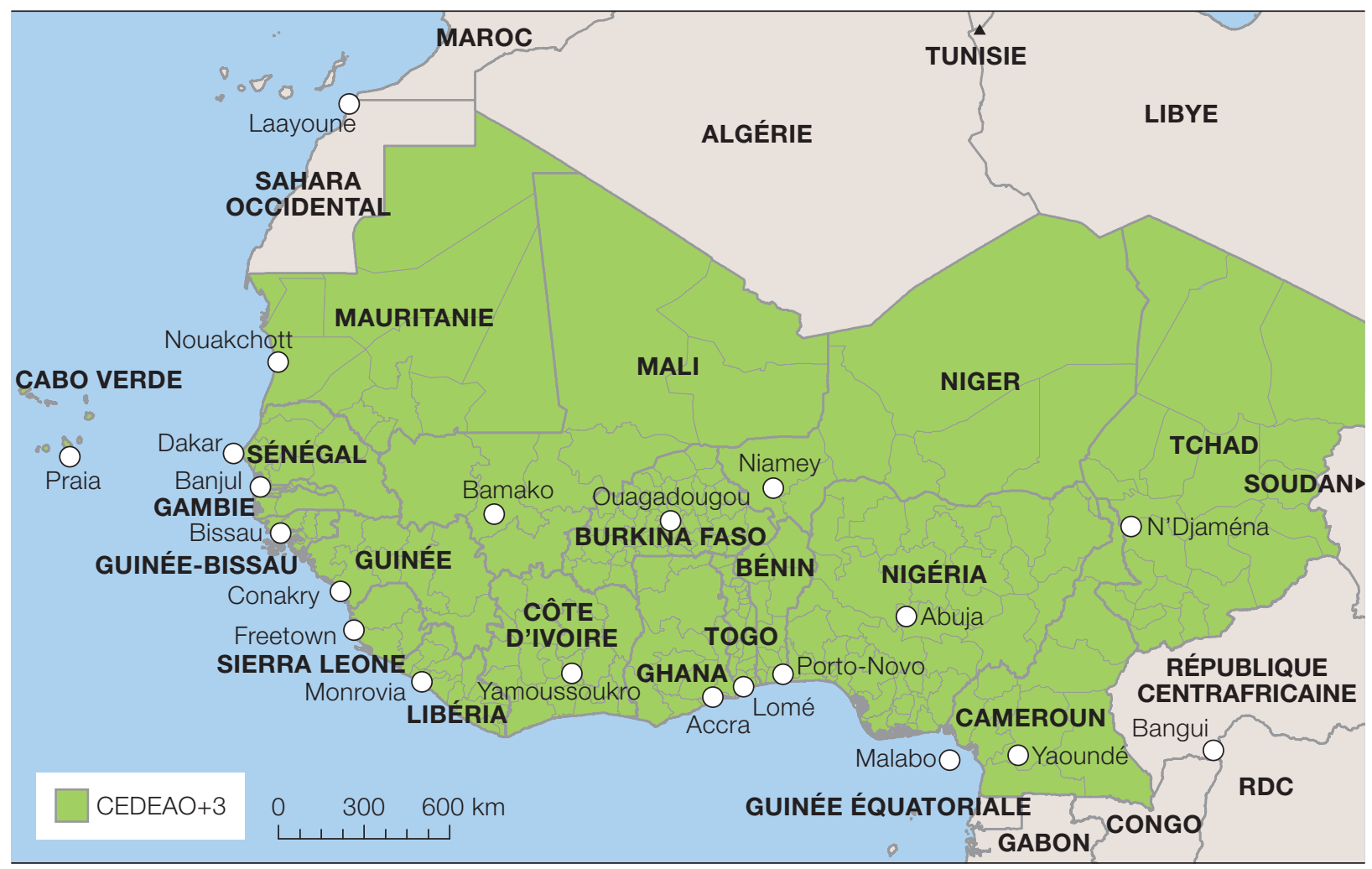

Source: OpenStreetMap

constitue un préalable à la mise en place de politiques de développement territorialisées, adaptées aux potentiels et contraintes des espaces urbains ouest-africains.

\section{Densité, distance et division}

Les villes frontalières d'Afrique de l'Ouest constituent un laboratoire d'analyse privilégié du processus d'intégration régionale. Leurs caractéristiques urbaines et leur proximité aux frontières d'État permettent de mettre en lumière trois dimensions fondamentales de l'intégration régionale: la densité, la distance et la division (Graphique 1.1). Ces 3 ( ( D ) correspondent chacune à un niveau géographique privilégié (Banque mondiale, 2009).

- Densité. Au niveau local, les villes frontalières forment un environnement dense dans lequel interagissent marchands, agriculteurs, fonctionnaires, autorités religieuses et représentants de la société civile, parfois séparés par des réglementations, des cultures, des langues et des monnaies différentes. La présence de compétences spécialisées et complémentaires en milieu urbain génère des avantages particuliers pour ceux qui y résident ou y font des affaires. Ces économies d'agglomération, présentes dans toutes les villes, sont particulièrement importantes en milieu frontalier 
dans la mesure où l'existence d'une frontière représente une ressource pouvant être mise à profit par le commerce ou la coopération.

- Distance. Au niveau national, nombre de villes frontalières sont situées à très grande distance des centres de décision. John Igué (1989) avait appelé ces espaces des (cpériphéries nationales)) pour attirer l'attention sur le développement de normes et de pratiques clientélistes autonomes par rapport à l'espace national. L'éloignement des villes frontalières aux centres de pouvoir est bien souvent synonyme de marginalisation politique. Il se manifeste notamment par un manque d'investissements publics en matière d'infrastructures routières, de services de santé ou d'infrastructures éducatives. Cette situation n'est pas sans poser de graves problèmes de cohésion nationale. Les mouvements autonomistes ou terroristes se nourrissent en effet souvent de la marginalisation - effective ou supposée - des espaces frontaliers pour diffuser leurs idées auprès des populations locales, comme dans le nord du Mali ou le nord-est du Nigéria depuis le milieu des années 2000.

- Division. Au niveau international, les villes frontalières servent de nœuds dans les réseaux de transport et de commerce qui relient les pays

Graphique 1.1

Densité, distance et division

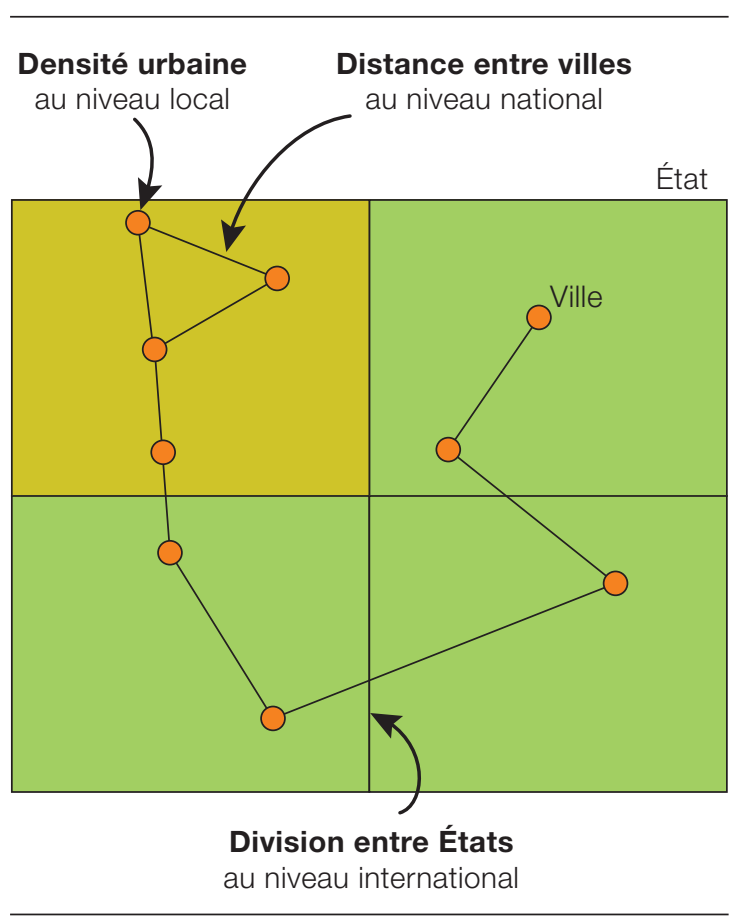
ouest-africains entre eux et les connectent aux marchés mondiaux. Leur rôle de carrefour permet de faire circuler des personnes et des produits qui ne trouveraient pas de débouchés au niveau local ou national. Il conduit au développement d'infrastructures de transport et de communication destinées à favoriser la fluidité des échanges régionaux, tels que des ponts, des embarcadères, des hangars de stockage ou des postes de contrôle gérés indépendamment ou conjointement par les États.

\section{Quelles sont les spécificités des villes frontalières?}

La spécificité des villes frontalières est étudiée à travers six indicateurs spécialement développés pour cette étude qui se rapportent à l'une des trois dimensions fondamentales de l'intégration régionale que sont la densité locale, la distance au plan national et la division internationale (Tableau 1.1). 
L'étude de l'évolution démographique et morphologique (Notes ouestafricaines $n^{\circ}$ 21) permet tout d'abord de caractériser les effets de la densité sur les villes frontalières au niveau local. Elle montre que la croissance des villes frontalières a été presque toujours supérieure à celle des autres

Tableau 1.1

Indicateurs et dimensions de l'intégration régionale

\begin{tabular}{l|l|l}
\hline $\begin{array}{l}\text { Densité } \\
\text { urbaine locale }\end{array}$ & $\begin{array}{l}\text { Distance entre } \\
\text { villes au plan } \\
\text { national }\end{array}$ & $\begin{array}{l}\text { Divisions entre } \\
\text { États au plan } \\
\text { international }\end{array}$ \\
\hline Démographie & Santé & Accessibilité \\
\hline Morphologie & Entreprises & Infrastructures \\
\hline
\end{tabular}
villes de la région depuis le milieu du $\mathrm{XX}^{\mathrm{e}}$ siècle. Cette croissance rapide est particulièrement visible à moins de 50 kilomètres des frontières nationales, où sont localisés les marchés les plus dynamiques. Particulièrement forte sur les frontières du Nigéria et dans le golfe de Guinée entre Bénin et Togo, elle est nettement moins marquée dans l'ouest de la région. En 2020, plus de 33 millions d'urbains habiteront dans ces villes, contre un peu plus de

20 millions en 2010. En conséquence, la part de la population ouest-africaine vivant dans les villes frontalières est en constante augmentation et atteindra $16 \%$ de la population urbaine totale à $50 \mathrm{~km}$ des frontières et $30 \%$ à $100 \mathrm{~km}$ à la fin de la décennie.

Du point de vue morphologique, les villes frontalières possèdent des spécificités bien particulières. Vingt-sept d'entre elles forment des agglomérations transfrontalières séparées par des frontières terrestres, par des fleuves ou situées le long d'un littoral. Contrairement aux apparences, les centres nationaux qui composent ces agglomérations ont bien souvent été dupliqués ou relocalisés après la partition coloniale, plutôt que séparés par les frontières actuelles (Soi et Nugent, 2017). Ces agglomérations transfrontalières ont pour particularités d'avoir émergé sans schéma d'aménagement concerté et demeurent très dépendantes les unes des autres. La récession actuelle qui frappe les citadins nigérians, par exemple, affecte très directement les vendeurs de voitures d'occasion de Cotonou au Bénin. La taille et les fonctions des centres de ces agglomérations transfrontalières varient grandement, particulièrement lorsqu'une capitale politique fait face à un autre centre urbain. N’Djaména au Tchad est ainsi plus de 20 fois plus peuplée que Kousséri au Cameroun (Carte 1.2) et Lomé plus de 11 fois plus grande qu'Aflao au Ghana.

Les effets de la distance sur les villes frontalières au plan national sont étudiés par le biais des services de santé et des entreprises formelles, dont l'implantation obéit très largement à des stratégies nationales (Notes ouest-africaines $n^{\circ}$ 22). La cartographie de la couverture sanitaire régionale met en évidence la profonde hétérogénéité des espaces frontaliers ouest-africains et le potentiel encore largement inexploité d'harmonisation des politiques de santé entre pays. De ce point de vue, le centre de santé partagé de Warokuy-Wanian, 


\section{Carte 1.2}

L'agglomération transfrontalière de N'Djaména-Kousséri (Tchad-Cameroun)

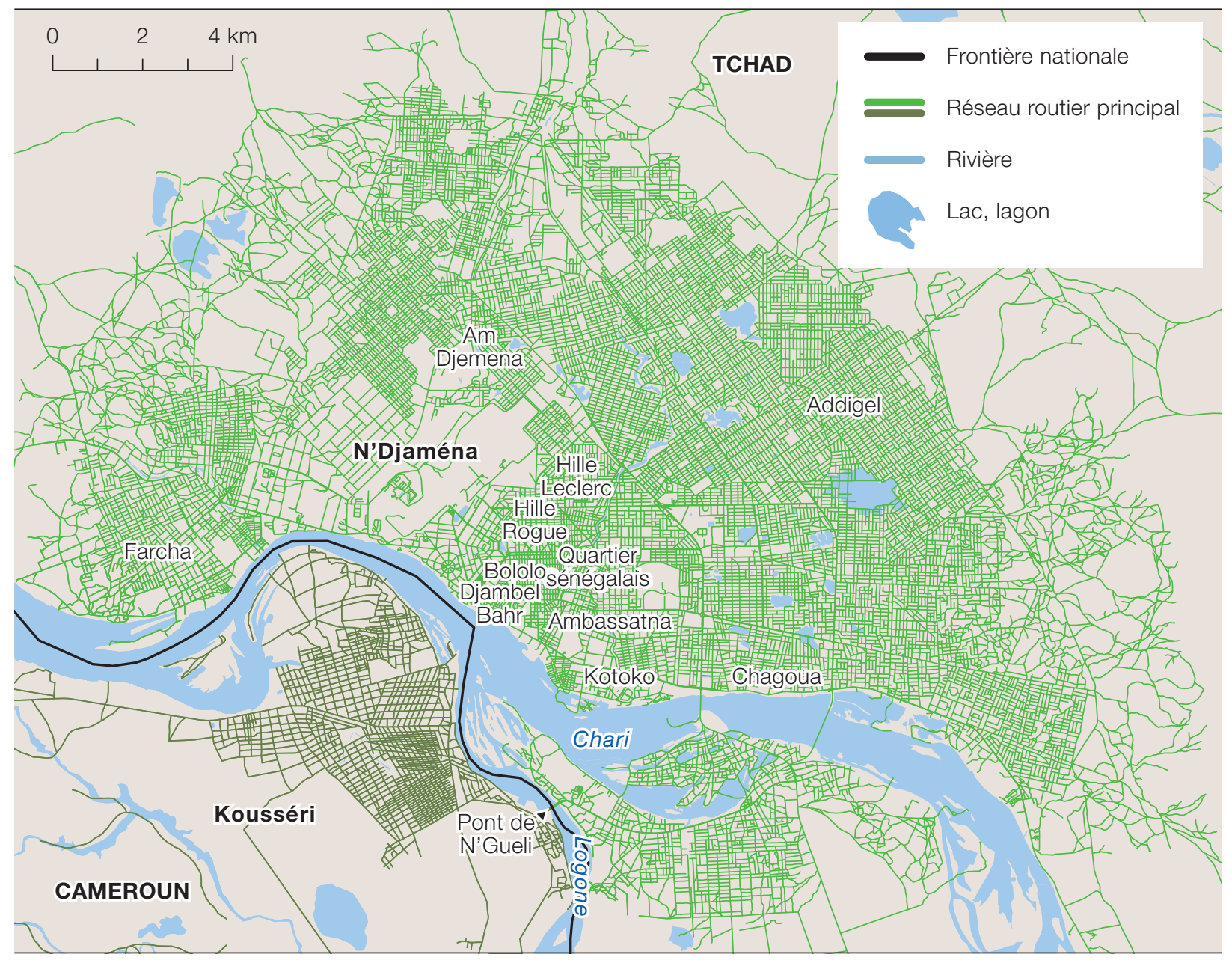

Source: OpenStreetMap 2018

souvent cité comme un exemple de coopération transfrontalière, apparaît comme une exception dans la région. La cartographie des entreprises formelles spécialisées dans certains secteurs stratégiques pour l'intégration régionale révèle une très forte concentration dans les capitales politiques et/ou économiques où se décident les politiques douanières, les stratégies d'importation et de réexportation et où sont localisées les plus grandes infrastructures de transport et de communication.

Au plan international, les effets de la division territoriale sont appréhendés par un modèle d'accessibilité spécialement développé pour rendre compte des effets de frontière sur les échanges régionaux (Notes ouest-africaines nº 23). Le modèle permet de calculer combien de personnes peuvent être atteintes à partir de chacune des villes frontalières de la région compte tenu de l'étendue du réseau routier, des vitesses moyennes observées et des contrôles routiers susceptibles de ralentir les échanges commerciaux sur les axes principaux et de renchérir le transport. Il évalue très précisément le gain d'accessibilité qui 
résulterait de l'ouverture effective des frontières à l'échelle régionale, de l'amélioration des conditions du réseau routier et de l'arrêt des contrôles policiers. Les résultats montrent que le bassin de population des villes frontalières pourrait être $14 \%$ plus important si le passage des frontières n'occasionnait aucune attente aux flux de biens et de personnes et de $12 \%$ plus important sans contrôle routier. Pour certains centres situés sur le golfe de Guinée, la multiplication des contrôles diminue l'accessibilité routière de plus de moitié. Entre Bénin, Niger et Nigéria, la prise en compte de l'état du réseau routier montre que le bassin de population combiné des huit villes frontalières augmenterait d'un tiers en l'absence d'attente aux frontières.

Cette analyse est complétée par une recension exhaustive des postes de contrôle juxtaposés mis en œuvre ou projetés par les États et les organisations régionales à l'échelle de l'Afrique subsaharienne. Elle révèle que la facilitation du commerce se heurte aux intérêts particuliers des fonctionnaires et des acteurs privés vivant des frictions de l'intégration régionale. En Afrique de l'Ouest en particulier, peu d'États sont aujourd'hui capables de tirer avantage de ces nouvelles structures de contrôle construites dans la région, dont la plupart sont non fonctionnelles.

\section{Quels obstacles au développement urbain transfrontalier?}

Le développement des villes frontalières est rendu difficile par une combinaison d'obstacles fonctionnels et institutionnels qui renforcent leur marginalisation.

Du point de vue socio-économique, les villes frontalières font face à un déficit d'infrastructures leur permettant de se développer à la fois comme centres d'innovation locaux et comme carrefours commerciaux régionaux. Cette double contrainte s'exprime localement par un développement urbain très largement spontané et par une insuffisance des marchés, zones de stockage, voies de communication urbaines et structures médico-sociales et éducatives. Du point de vue des échanges régionaux, les villes frontalières souffrent de l'engorgement et du manque d'entretien des infrastructures routières et ferroviaires qui les relient au reste du pays et aux pays voisins. Le manque d'investissements productifs, socio-éducatifs et marchands, caractéristique des villes frontalières, réduit considérablement les économies d'agglomérations potentielles qui résultent de la concentration urbaine. Il renforce en outre les effets négatifs de la distance au plan national et impose de lourdes contraintes aux échanges régionaux, contribuant à faire de l'Afrique subsaharienne l'un des endroits du monde les plus chers pour faire du commerce (Teravaninthorn et Raballand, 2009).

Du point de vue institutionnel, le principal obstacle au développement des villes frontalières réside dans la faible capacité financière des collectivités territoriales. Bien que la décentralisation ait atteint un niveau de traduction 
satisfaisant sur le plan juridique et institutionnel au sein de la région, avec l'existence de schémas décentralisateurs dans l'ensemble des pays, la mise à disposition des outils et des moyens financiers nécessaires à la réalisation concrète de ces ambitions reste encore limitée. La faible autonomie fiscale, le taux de recouvrement limité et la tendance à ne pas augmenter les impôts en période électorale aggravent cette situation. En conséquence, les collectivités locales manquent souvent de moyens pour mettre en place des investissements décentralisés qui permettraient d'exploiter leurs potentialités urbaines et frontalières.

\section{Quelles politiques de développement pour les villes frontalières?}

L'importance démographique et économique des villes frontalières pour l'intégration régionale justifie que des politiques de développement adaptées à leurs spécificités soient mises en œuvre par les États et leurs partenaires régionaux et internationaux. Ces politiques territorialisées doivent accompagner la croissance urbaine et la formation d'espaces urbains transfrontaliers en développant des infrastructures et des biens publics adaptés à différentes échelles (OCDE, 2009; OCDE/CSAO, 2017).

- Les politiques de développement doivent favoriser les avantages résultant de la densité urbaine au niveau local en appuyant le développement de plans d'aménagement qui maximisent les interactions intra-urbaines. Les économies d'agglomération qui résultent des fortes densités urbaines permettent potentiellement de minimiser les coûts de transport et de communication, de faciliter la diffusion d'innovations et de rendre plus accessibles certains produits agricoles ou manufacturés. Elles sont cependant plus facilement exploitables par les acteurs si les villes se développent de manière planifiée et concertée avec les villes frontalières voisines. La croissance rapide de la population et l'étalement urbain actuellement observés dans les villes frontalières d'Afrique de l'Ouest devraient inciter les États et leurs partenaires à investir durablement dans l'accompagnement de ces dynamiques s'ils souhaitent tirer bénéfice des économies d'agglomérations.

- Au plan national, l'enjeu des politiques de développement est de réduire la distance qui sépare les villes frontalières des autres nœuds du réseau urbain afin de minimiser les inconvénients liés à leur marginalisation géographique et de favoriser la cohésion nationale. De ce point de vue, le développement des villes frontalières est indissociable des politiques nationales visant à projeter l'autorité et les services de l'État sur l'ensemble du territoire national.

- Au niveau international, les politiques de développement doivent continuer à réduire les frictions qui résultent des 32000 kilomètres de frontières terrestres qui divisent les États d'Afrique de l'Ouest. Cet objectif peut être 
atteint en facilitant la mobilité des biens, des personnes, du capital et des idées à courte et longue distance. Les corridors de transport, les ports secs, les postes de contrôle juxtaposés, les zones de libre-échange et les pipelines actuellement implémentés par les organisations régionales et les États, doivent être soutenus par les politiques de développement.

Du point de vue fonctionnel, l'une des difficultés majeures des politiques de développement destinées aux villes frontalières est de tenir compte des intérêts divergents des acteurs qui y résident, y transitent, ou y commercent à distance. Les villes frontalières servent en effet de carrefour à la fois aux populations locales qui traversent les frontières au quotidien pour commercer ou socialiser, aux marchands qui animent le commerce régional par la route et le rail, et aux grands opérateurs économiques actifs dans le commerce des matières premières et des produits manufacturés à partir des villes capitales (Dobler, 2016).

Tandis que les populations locales et les marchands régionaux vivent en partie du maintien des différentiels de prix et de taxation entre pays, les grands opérateurs, qui réalisent l'essentiel de leur profit à l'échelle mondiale, ont intérêt à une harmonisation du commerce régional. Ces opérateurs sont les plus fervents partisans des initiatives destinées à supprimer les frictions liées au commerce international (Zeller, 2008). Leurs intérêts rejoignent ceux des organisations régionales, qui promeuvent ces infrastructures, tandis que les marchands régionaux et les fonctionnaires d'État chargés du contrôle des frontières, qui ont souvent tissé des réseaux clientélistes, y sont généralement opposés.

Les politiques de développement doivent tenir compte du fait que les villes frontalières constituent tout à la fois un espace de vie pour les populations locales, un carrefour obligé pour les marchands et une étape coûteuse pour les grands opérateurs mondiaux. En conséquence, ces politiques de développement ne peuvent se limiter à la promotion de zones de libre-échange et de corridors de transport régionaux dont l'intérêt se porte essentiellement sur le commerce formel entre centres urbains au détriment des conséquences pour le développement local et l'économie informelle.

Du point de vue institutionnel, le développement des villes frontalières passe par une politique volontariste de décentralisation financière. La période actuelle est favorable à ce type d'évolution. En effet, grâce au plaidoyer du Conseil des collectivités territoriales (CCT) de l'Union économique et monétaire ouest-africaine (UEMOA), le Programme de coopération transfrontalière locale (PCTL) incite actuellement les institutions régionales et les États à intégrer davantage la coopération transfrontalière locale dans leurs politiques publiques. Cette évolution peut bénéficier aux villes frontalières. Plusieurs schémas d'aménagement transfrontaliers intégrés (SATI) ont été financés pour favoriser les possibilités de coopération transfrontalière entre 
collectivités locales du Bénin, du Burkina Faso, de la Côte d'Ivoire, du Mali et du Niger. Ce nouvel outil de coopération entre opérateurs sectoriels, acteurs régionaux et transfrontaliers doit permettre de fixer des objectifs, d'établir des fiches de projets transfrontaliers locaux et de convaincre les bailleurs de financer les projets. Parmi les projets prioritaires transfrontaliers portés par les SATI en Afrique de l'Ouest figurent le désenclavement, le développement économique et social ainsi que la gouvernance. Les politiques de développement doivent accompagner ce processus et travailler à un renforcement du poids politique des villes et des régions dans les processus de décision qui affectent directement les échanges aux frontières. 

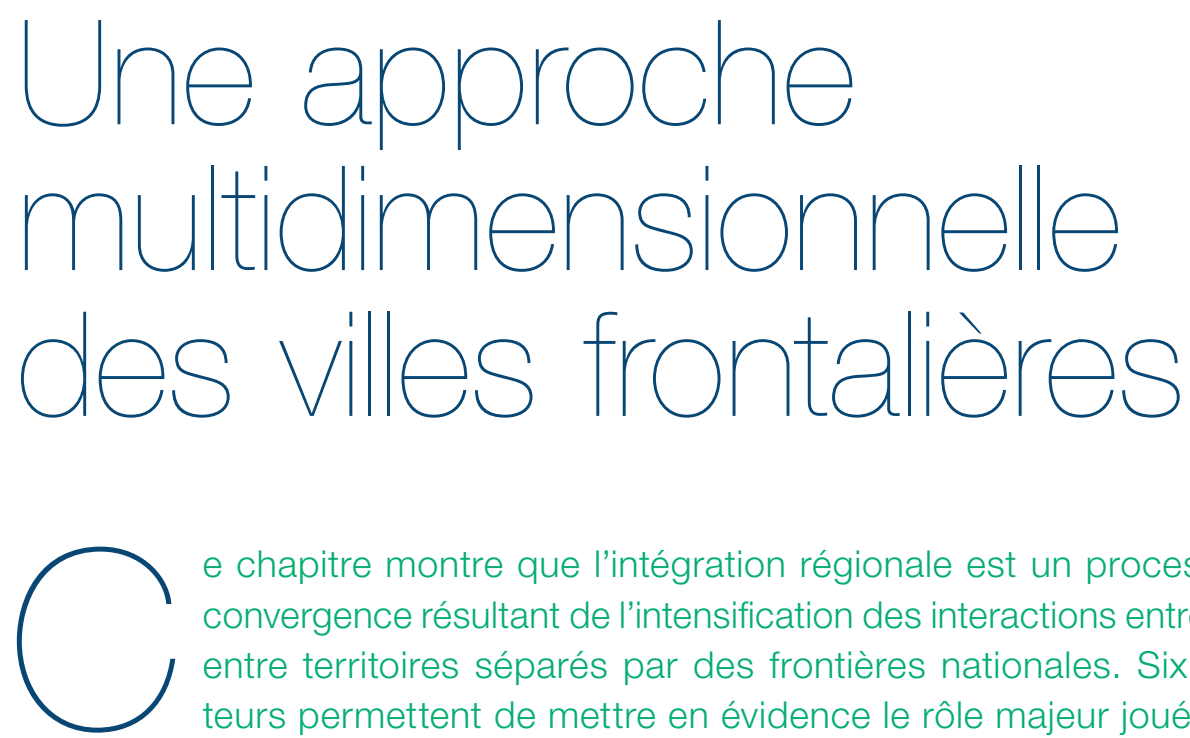

e chapitre montre que l'intégration régionale est un processus de convergence résultant de l'intensification des interactions entretenues entre territoires séparés par des frontières nationales. Six indicateurs permettent de mettre en évidence le rôle majeur joué par les villes frontalières dans ce processus aussi bien au niveau local que national et international: démographie, morphologie urbaine, entreprises formelles, infrastructures de santé, accessibilité routière, postes de contrôle frontaliers. Il présente la manière dont ces indicateurs sont sélectionnés, les sources utilisées pour les construire et leur contribution à l'étude multidimensionnelle des villes frontalières.

\section{Convergence et interactions}

L'intégration régionale peut prendre deux formes complémentaires. Il s'agit d'une part d'un processus de convergence entre territoires nationaux, dont les caractéristiques physiques et humaines tendent à s'homogénéiser. Du fait de l'intégration régionale, par exemple, les différences dans la morphologie des agglomérations urbaines ou le niveau de vie des populations peuvent tendre à se réduire au cours du temps. Comme le montre le Graphique 2.1, deux agglomérations se développant de part et d'autre d'une frontière peuvent connaître des densités décroissantes à partir de leur noyau urbain. Au cours du processus d'intégration, ces densités sont susceptibles de converger jusqu'à former un seul ensemble urbain.

D'autre part, l'intégration régionale constitue un processus d'intensification des interactions sociales, économiques et politiques entre acteurs situés dans différents pays. Les espaces frontaliers sont ainsi davantage intégrés s'ils sont connectés par des flux de personnes, de marchandises ou par des accords de coopération. Ce processus est illustré par le Graphique 2.1, qui montre comment la création de nouvelles routes entre villes frontalières, représentées en rouge, est susceptible de favoriser les échanges socio-économiques et politiques. 
Graphique 2.1

Convergence et interactions dans le processus d'intégration

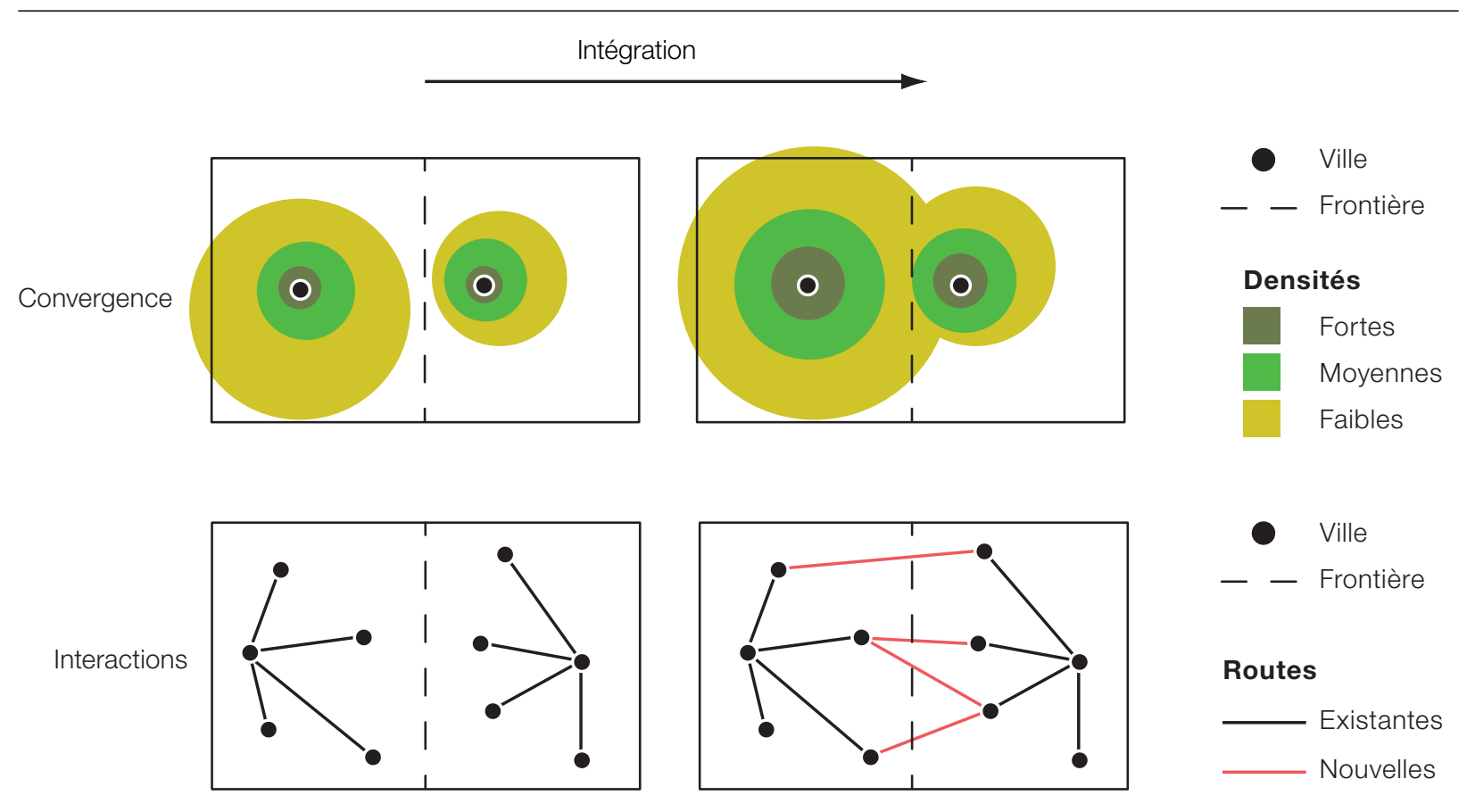

Source: adapté de De Boe et al. 1999

L'étude combinée de la convergence et des interactions conduisant à l'intégration régionale pose trois enjeux particuliers d'ordre méthodologique, conceptuel et spatial.

Tout d'abord, convergence et interactions ne peuvent être mesurées de la même manière. En effet, si la convergence se mesure par l'intermédiaire des attributs des villes frontalières, comme la taille ou la densité, les interactions nécessitent que soient pris en considération les flux effectifs qui relient ces villes aux autres centres urbains. Or, en Afrique comme dans le reste du monde, les données se rapportant aux attributs des villes sont nettement plus nombreuses que celles touchant à leurs relations. Cette situation résulte du fait qu'il est souvent plus aisé de comptabiliser les attributs statiques des villes, comme leur surface ou leur population, que les flux humains et commerciaux, qui sont par définition dynamiques. Le manque de données relationnelles s'explique également par le fait que les organismes chargés de la collecte des statistiques privilégient les données territoriales portant sur des stocks visibles et quantifiables à l'échelle nationale plutôt que les interactions intra ou interurbaines (Taylor, 2013). Ce problème commun à tous les États prend des proportions encore plus importantes en Afrique subsaharienne, où les données relationnelles à l'échelle subnationale sont particulièrement rares, même dans des domaines pourtant cruciaux pour le développement urbain, comme les migrations ou les déplacements domicile-travail quotidiens. 
À ces difficultés d'ordre statistique s'ajoute un paradoxe. En effet, les études conduites dans le domaine de l'intégration régionale tendent à montrer que l'accroissement des interactions entre villes et territoires frontaliers ne favorise pas nécessairement leur convergence (Sohn et al., 2009; Decoville et al., 2013). Autrement dit, l'intégration régionale se nourrit des différences entre espaces séparés par des frontières nationales, ce qui implique que les espaces fortement connectés sont aussi ceux où sont observées les plus grandes disparités. Ce paradoxe s'explique tout d'abord par le fait que les relations entre villes sont souvent très inégales et conduisent à de forts différentiels de revenus ou de croissance économique. Certaines villes se spécialisent dans le commerce de certains biens, l'offre résidentielle ou le développement industriel, en fonction de leurs avantages comparatifs. De fortes relations fonctionnelles ne sont ainsi pas une garantie de développement commun. D'autre part, un processus de convergence peut résulter de dynamiques internes aux villes et régions frontalières sans que de fortes interactions les relient. Deux États peuvent ainsi mettre en œuvre des politiques de décentralisation qui aboutissent à renforcer l'autonomie des villes frontalières sans nécessairement que celles-ci renforcent leurs relations mutuelles.

Enfin, le processus d'intégration régionale appréhendé au travers des villes frontalières mobilise trois échelles géographiques bien particulières (Banque mondiale, 2009). Les villes frontalières sont en effet des environnements urbains denses qui facilitent les échanges locaux et fournissent des opportunités à ceux qui y habitent ou les fréquentent. Ces économies d'agglomérations sont favorisées par la proximité de la frontière, qui fonctionne bien plus comme une ressource que comme une barrière entre villes frontalières d'Afrique de l'Ouest. Les villes frontalières sont également des points d'entrée et de sortie obligés pour les biens et les services qui alimentent le réseau urbain national. La distance qui les sépare du reste du territoire national est susceptible d'affecter la cohésion nationale et d'alimenter, en cas de marginalisation, les mouvements autonomistes ou indépendantistes dans les zones frontalières. Finalement, les villes frontalières forment des nœuds dans les échanges internationaux. Par leurs infrastructures et leur position stratégique, elles sont des relais qui peuvent faciliter ou, au contraire, renforcer les effets de la division territoriale de l'Afrique de l'Ouest.

\section{Une analyse multidimensionnelle}

Les particularités du processus d'intégration nécessitent que soit conduite une analyse multidimensionnelle (six indicateurs) qui tienne compte à la fois des attributs des villes, de leurs relations et des différents niveaux spatiaux (Tableau 2.1, Encadré 2.1).

Ces six indicateurs sont loin de couvrir l'ensemble des dimensions possibles du processus d'intégration. En outre, du fait de l'absence de données infra- 
nationales portant sur les flux transfrontaliers de pendulaires, les différentiels de richesse per capita ou la proportion de résidents étrangers, habituellement utilisés pour mesurer le processus d'intégration régionale, les indicateurs retenus sont moins complexes que ceux développés dans la littérature consacrée aux villes frontalières européennes (ESPON, 2010; Decoville et al., 2013) ou aux villes globales (Taylor et Derudder, 2016). Seule l'accessibilité routière peut être qualifiée d'indicateur d'interactions, les autres mesurant les attributs des villes.

Le seuil à partir duquel les dynamiques frontalières influencent les villes ouest-africaines est susceptible de changer selon les indicateurs considérés. L'étude de la démographie est conduite aux seuils de 20, 50 et 100 kilomètres. Pour les établissements de santé, un seuil de $20 \mathrm{~km}$ est plus adapté à rendre compte des déplacements transfrontaliers éventuels des populations, d'autant plus que seuls les établissements de type intermédiaire et supérieur comme les hôpitaux et les centres régionaux de santé, sont retenus. On estime qu'un malade devant se faire soigner dans ce genre d'établissement sera désireux de parcourir une distance assez importante (jusqu'à $40 \mathrm{~km}$ aller-retour à vol d'oiseau). Un seuil de $50 \mathrm{~km}$ est considéré pour les entreprises formelles, dont les sièges et bureaux principaux sont souvent installés à une certaine distance des frontières. Ce seuil permet d'inclure certaines des villes principales où sont installées les entreprises d'envergure régionale et non pas seulement les marchés frontaliers.

Seules les villes situées à moins de 20 km d'une frontière terrestre sont prises en considération dans l'étude de la morphologie et de la division. Ce choix

Tableau 2.1

Indicateurs relatifs aux villes frontalières

\begin{tabular}{|c|c|c|c|}
\hline Dimension & Indicateurs & Définition & Seuils \\
\hline \multirow[t]{2}{*}{ Densité locale } & Population & $\begin{array}{l}\text { Population, densité et croissance des villes fronta- } \\
\text { lières. }\end{array}$ & $\begin{array}{l}20,50, \\
100 \mathrm{~km}\end{array}$ \\
\hline & Morphologie & $\begin{array}{l}\text { Bâti urbain et existence d'une agglomération morpho- } \\
\text { logique transfrontalière. }\end{array}$ & $20 \mathrm{~km}$ \\
\hline \multirow[t]{2}{*}{$\begin{array}{l}\text { Distance } \\
\text { nationale }\end{array}$} & Entreprises & $\begin{array}{l}\text { Entreprises formelles spécialisées dans certains } \\
\text { secteurs importants pour l'intégration régionale. }\end{array}$ & $50 \mathrm{~km}$ \\
\hline & Santé & Infrastructures de santé d'envergure transfrontalière. & $20 \mathrm{~km}$ \\
\hline \multirow[t]{2}{*}{$\begin{array}{l}\text { Division } \\
\text { internationale }\end{array}$} & $\begin{array}{l}\text { Accessibilité } \\
\text { routière }\end{array}$ & $\begin{array}{l}\text { Nombre de personnes pouvant potentiellement être } \\
\text { atteintes depuis les villes frontalières en moins de } \\
4 \text { heures. }\end{array}$ & $20 \mathrm{~km}$ \\
\hline & Infrastructures & Postes de contrôle juxtaposés. & $20 \mathrm{~km}$ \\
\hline
\end{tabular}


méthodologique permet de limiter l'analyse aux villes dont les agglomérations, l'accessibilité et les infrastructures sont les plus susceptibles d'être influencées par une frontière. Il assure en outre la possibilité de comparer les résultats de cette étude avec ceux des travaux plus anciens consacrés aux villes et marchés frontaliers ouest-africains qui avaient aussi utilisé ce seuil (OCDE/CSAO, 2017, 2014).

\section{Six indicateurs d'intégration}

\section{Densité}

Les effets locaux de la densité sont appréhendés au travers de la population et de la morphologie des villes frontalières (Encadré 2.1 pour les sources).

- L'étude démographique permet de caractériser la taille, la densité et la croissance des villes frontalières ouest-africaines depuis les années 50. L'utilisation de la base de données harmonisée Africapolis garantit que les résultats sont comparables entre tous les pays de la région. Cette étude permet en outre de comparer l'évolution démographique des villes frontalières à celle des autres villes du réseau urbain, de manière à mettre en évidence les spécificités frontalières.

- Létude de la morphologie des villes frontalières se focalise sur les agglomérations transfrontalières, définies comme des unités urbaines formant un tissu continu de part et d'autre des frontières. Dans ces ensembles urbains particulièrement interdépendants, elle permet de montrer de quelle manière l'existence d'une frontière terrestre ou fluviale affecte la continuité du bâti.

\section{Distance}

Deux indicateurs permettent de considérer les effets de la distance sur les villes frontalières : les établissements de santé et les entreprises formelles. Ils possèdent la particularité d'obéir à des dynamiques nationales. L'implantation des établissements de santé répond en effet à des (( cartes sanitaires )) visant à atteindre le plus grand nombre de patients dans la mesure des ressources disponibles. La distribution géographique des entreprises formelles considérées dans ce rapport répond également à des impératifs nationaux. Il s'agit de desservir au mieux le marché de chacun des États de l'Afrique de l'Ouest. L'étude de ces indicateurs permet alors de montrer dans quelle mesure les villes frontalières participent à la cohésion nationale.

- L'étude des entreprises se concentre sur celles spécialisées dans certains secteurs vitaux pour l'intégration régionale, comme la logistique et le transit, le transport routier et ferroviaire de marchandises, les services maritimes et le commerce international. Les entreprises considérées possèdent un siège social, sont enregistrées dans le registre des entreprises nationales et s'acquittent de taxes. Les entreprises informelles 
actives dans le commerce transfrontalier, particulièrement nombreuses dans les régions frontalières ouest-africaines, ne sont pas comptabilisées du fait de l'impossibilité de recenser leurs activités.

- L'analyse du secteur de la santé recense et localise les infrastructures de soin d'envergure transfrontalière en Afrique de l'Ouest. L'indicateur se concentre sur les établissements de soin présentant un potentiel d'attraction suffisant pour motiver une coopération institutionnelle entre deux entités géographiques séparées par une frontière nationale ou le déplacement transfrontalier des populations locales. Cet indicateur inédit permet de mettre en évidence des différentiels dans la couverture sanitaire nationale et transfrontalière.

\section{Division}

Les effets de la division internationale sont pris en considération par le biais d'un modèle d'accessibilité et d'une étude des postes de contrôle frontaliers juxtaposés. Ces indicateurs permettent de rendre compte des interactions qui relient les villes frontalières au reste de l'Afrique de l'Ouest.

- L'indicateur d'accessibilité cartographie le bassin de population pouvant théoriquement être atteint en moins de 4 heures de route à partir de n'importe quelle ville frontalière de plus de 10000 habitants en 2015. Initialement développé pour calculer le potentiel de population des régions européennes par l'Institut Alterra de l'Université de Wageningen (van Eupen et al., 2012; Gløersen, 2012; Jochem, 2016), le modèle a été tout d'abord appliqué aux cas des marchés frontaliers ouest-africains par le Secrétariat du CSAO (OCDE/CSAO, 2017) avant d'être généralisé à l'ensemble des villes frontalières de la région dans la présente étude. Le modèle permet de recréer les conditions réelles de déplacement dans la région en tenant compte à la fois de l'étendue, de l'existence de contrôles routiers et d'autres variables physiques qui affectent la vitesse moyenne des véhicules dans la région, comme la pente, la végétation, ou l'existence de fleuves et de frontières. Dans la région du Dendi, entre Bénin, Niger et Nigéria, l'état effectif du réseau routier est pris en compte.

- Les données relatives aux postes de contrôle juxtaposés permettent de géolocaliser 59 postes à l'échelle de l'Afrique subsaharienne. L'indicateur renseigne sur leur statut en 2017-18 selon les catégories suivantes : planifié, en construction, inauguré sans être opérationnel et fonctionnel. 


\section{Encadré 2.1}

Source des données

Démographie et morphologie. Base de données harmonisée Africapolis, qui recense l'ensemble des agglomérations urbaines de plus de 10000 habitants de 1950 à 2015 (OCDE/CSA0, 2018) et images satellitaires Google Maps et Google Earth.

Santé. Global Healthsites Mapping Project de la plateforme ouverte Humanitarian Data Exchange (HDX) du Bureau de la coordination des affaires humanitaires des Nations Unies (UNOCHA). Les données utilisées concernent l'année 2017 ou plus proche et sont collectées par les États, les organisations internationales ou les organismes non gouvernementaux. Dans la région de Diffa au Niger, les données collectées depuis 2013 par le Haut-Commissariat aux réfugiés des Nations Unies (UNHCR) et REACH ont été utilisées en complément. Dans le Liptako-Gourma, les données du Fonds d'équipement des Nations Unies (FENU), du Programme des Nations Unies pour le développement (PNUD) et du programme LOBI du FENU collectées dans le cadre de leur Annuaire cartographique de l'espace transfrontalier Mali-Burkina-Niger ont été mobilisées. Au Tchad (2013), au Burkina Faso (2013), au Niger (2014) et au Mali (2012), les annuaires statistiques sanitaires nationaux ont été utilisées en complément des données HDX.

Entreprises. Africa Directory pour le Bénin, le Burkina Faso, le Cabo Verde, le Ghana, le Nigéria, le Sénégal et le Tchad, et Go Africa pour la Côte d'Ivoire, la Guinée, la Guinée-Bissau, le Libéria, la Mauritanie, le Niger, la Sierra Leone et le Togo. Les données utilisées concernent l'année 2017 ou plus proche. D'autres sources ont été utilisées en complément: le répertoire du Ministère du commerce de Gambie, Afrique pages jaunes et Delili pour la Mauritanie, Business Sierra Leone, DCLA et la Chambre de commerce de Sierra Leone, Buyers Directory pour le Libéria, la Sierra Leone et la Guinée-Bissau, Agence pour la promotion des investissements pour le Mali et Annuaire des entreprises 2016 pour le Niger.

Accessibilité. Couverture terrestre de l'Agence spatiale européenne (European Space Agency, 2010), réseaux routiers d'OpenStreetMap (2018) et du Global Roads Open Access Data Set (2018), altitudes de la NASA (2014), frontières de Global Administrative Areas (2018), population de LandScan TM (2014) et Africapolis (OCDE/ CSA0, 2018). Les données du Comité permanent interÉtats de lutte contre la sécheresse dans le Sahel (CILSS, 2017) sur les tracasseries routières permettent d'affiner le modèle en localisant les postes de contrôle sur les corridors de transport principaux en septembre 2017. Les vitesses moyennes sont estimées à $30 \mathrm{~km}$ par heure sur l'ensemble du réseau, sauf dans la région du Dendi, entre Bénin, Niger et Nigéria où des données de terrain collectées par l'Université de Niamey permettent de travailler sur des vitesses tenant compte de l'état réel des routes.

Infrastructures. Enquête auprès d'experts internationaux, complétée par une analyse d'images satellitaires Google Maps, d'observations de terrain et de sources bibliographiques. 


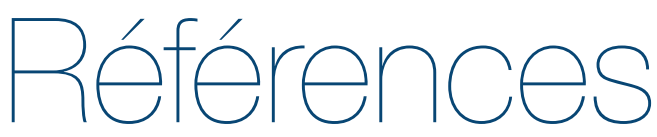

Agence spatiale européenne (2010), «Land Cover », ESA, Paris, www.esa-landcover-cci.org.

Banque mondiale (2009), World Development Report, Reshaping Economic Geography, Banque mondiale, Washington, DC, https://doi.org/10.1787/9789264265974-fr.

CILSS (2017), Rapport mensuel sur les tracasseries routières des produits agropastoraux dans le Sahel et en Afrique de l'Ouest, septembre, Comité permanent inter-États de lutte contre la sécheresse dans le Sahel, Ouagadougou.

De Boe P., C. Grasland et A. Healy (1999), Spatial Integration, Study Programme on European Spatial Planning, Final report 1.4, Commission européenne, Bruxelles.

Decoville A., F. Durand, C. Sohn et O. Walther (2013), "Comparing cross-border metropolitan integration in Europe: Towards a functional typology ", Journal of Borderlands Studies, $n^{\circ} 28$, vol. 2, pp. 221-237.

Dobler, G. (2016), «The Green, the Grey and the Blue: A Typology of Cross-border Trade in Africa", The Journal of Modern African Studies, vol. 54, no 1, pp. 145-169, https://doi.org/10.1017/S0022278X15000993.

ESPON (2010), METROBORDER - Cross-border Polycentric Metropolitan Regions, European Spatial Planning Observation Network, Luxembourg.

Global Administrative Areas (2018), GADM database of Global Administrative Areas, GADM 2.8, www.gadm.org.

Global Roads Open Access Data Set (2018), Global Roads Open Access Data Set, Version 1 (gROADSv1), NASA Socioeconomic Data and Applications Center (SEDCAC); Center for International Earth Science Information Network (CIESIN), Columbia University; et Information Technology Outreach Services (ITOS), University of Georgia, https://doi.org/10.7927/H4VD6WCT.

Gløersen, E. (dir. pub.) (2012), GEOSPECS - Geographic Specificities and Development Potentials in Europe: European Perspective on Specific Types of Territories, Final Scientific Report, ESPON, Luxembourg.

Igué, J. (1989), "Le développement des périphéries nationales en Afrique», dans Tropiques. Lieux et Liens, Paris, ORSTOM, pp. 594-605.

Jochem, R. (2016), REPOScan - REgion POtential Scanner; a standalone tool to evaluate landscape potentials based on variations in the landscape impedance. Basic Tool description, Alterra, Wageningen University \& Research, Wageningen.

LandScan TM Global Population Database (2014), Global Population Database, Oak Ridge National Laboratory, Oak Ridge.

NASA (2014), Shuttle Radar Topography Mission, NASA, Jet Propulsion Laboratory, California Institute of Technology, Pasadena: http://www2.jpl.nasa.gov/srtm.

OCDE (2009), Régions et croissance: Une analyse des tendances, Éditions OCDE, Paris, http://dx.doi.org/10.1787/9789264056541-fr.

OCDE/CSAO (2019), Africapolis: Dynamiques d'urbanisation en Afrique, Cahiers de l'Afrique de l'Ouest, Éditions OCDE, Paris (à paraître).

OCDE/CSAO (2018), Africapolis (base de données), www.africapolis.org.

OCDE/CSAO (2017), Coopération transfrontalière et réseaux de gouvernance en Afrique de l'Ouest, Éditions OCDE, Paris.

OCDE/CSAO (2014), Un Atlas du Sahara-Sahel: Géographie, économie et insécurité, Éditions OCDE, Paris.

OpenStreetMap Foundation (2018), «Roads », OpenStreetMap, OpenStreetMap Foundation, Sutton Coldfield, www.openstreetmap.org.

Sohn, C., B. Reitel et O. Walther (2009), "Cross-border metropolitan integration in Europe: the case of Luxembourg, Basel and Geneva", Environment and Planning C: Government and Policy, n²7, pp. 922-939.

Soi, I. et P. Nugent (2017), «Peripheral Urbanism in Africa: Border Towns and Twin Towns in Africa", Journal of Borderlands Studies, vol. 32, n 4, pp. 535-556, https://doi.org/10.1080/08 865655.2016.1196601. 
Taylor, P. J. (2013), Extraordinary Cities: Millennia of Moral Syndromes, World-systems and City/ state Relations, Edward Elgar, Cheltenham.

Taylor, P. J. et B. Derudder (2016), World City Network: A Global Urban Analysis, Routledge, Londres.

Teravaninthorn, S. et G. Raballand (2009), Transport Prices and Costs in Africa. A Review of the International Corridors, Banque mondiale, Washington, DC.

Van Eupen, M., M.J. Metzger, M. Pérez-Soba et al. (2012), «A rural typology for strategic European policies ", Land Use Policy, n² 29, pp. 473-482.

Zeller, W. (2008), "Danger and Opportunity in Katima Mulilo: A Namibian Border Boomtown at Transnational Crossroads", Journal of Southern African Studies, vol. 35, n 1, pp. 133-154. 


\section{Notes ouest-africaines}

\section{Intégration régionale des villes frontalières}

Cette Note qui fait partie de la Collection «Villes», souligne la contribution des villes frontalières au processus d'intégration régionale en Afrique de l'Ouest. Six indicateurs permettent d'appréhender les dynamiques locales développées en milieu urbain, les effets de la distance sur la cohésion nationale, et l'impact des divisions territoriales au plan international dans 18 pays. Cette analyse met en évidence les obstacles qui limitent le potentiel d'intégration des villes frontalières du point de vue des échanges socio-économiques et des institutions. La Note conclut par une synthèse des politiques territorialisées pouvant être mises en place dans la région pour faciliter le développement économique et politique des villes frontalières ouest-africaines. 